\title{
Arthroscopic Pullout Fixation for a Small and Comminuted Avulsion Fracture of the Posterior Cruciate Ligament from the Tibia
}

\author{
Shuji Nakagawa, $\mathrm{MD}^{1}$, Yuji Arai, $\mathrm{MD}^{2}$, Kunio Hara, $\mathrm{MD}^{1,3}$, Hiroaki Inoue, $\mathrm{MD}^{1}$, Manabu Hino, $\mathrm{MD}^{1}$, and \\ Toshikazu Kubo, $\mathrm{MD}^{1}$ \\ Departments of ${ }^{1}$ Orthopaedics, and ${ }^{2}$ Sports and Para-Sports Medicine, Graduate School of Medical Science, Kyoto Prefectural University of Medicine, Kyoto; \\ ${ }^{3}$ Department of Orthopaedics Surgery, Social Insurance Kyoto Hospital, Kyoto, Japan
}

\begin{abstract}
We describe a patient who underwent arthroscopic pullout fixation for a posterior cruciate ligament (PCL) avulsion fracture. A 46-year-old female, injured in a fall while riding a motorcycle, was diagnosed with a right knee PCL tibial attachment avulsion fracture and underwent arthroscopic osteosynthesis. A Kirschner wire was drilled to a point just medial to the medial border of the anterior tibial bony bed. A suture wire was folded into a loop and introduced into the posteromedial compartment via the bone tunnel. A fixation thread was inserted from the posteromedial portal, through the medial and lateral loop wires, and into the posteromedial compartment. The lateral and medial loop wires attached to the thread were pulled to the outside, and the thread was fixed onto the tibia. Three months post-surgery, she returned to her job. This procedure represents a minimally invasive method of treating avulsion fractures of the tibial attachment of the PCL.
\end{abstract}

Keywords: Knee, Posterior cruciate ligament, Fracture, Avulsion, Arthroscopy, Fixation

Surgical reduction and fragment fixation have been shown superior to conservative therapy in the treatment of avulsion fractures of the posterior cruciate ligament (PCL) attachment to the tibia. Surgical treatment is recommended for PCL injuries with $\geq 10 \mathrm{~mm}$ posterior instability ${ }^{1)}$. Until recently, posterior approaches to the fracture site have commonly been used for surgical reattachment ${ }^{2}$. These approaches, however, require a large skin incision to avoid damage to the popliteal neurovascular structures located immediately behind the site. Arthroscopic methods are safer and less invasive than posterior repair approaches.

Received May 7, 2016; Revised (1st) June 28, 2016; (2nd) July 25, 2016; Accepted July 31, 2016

Correspondence to: Yuji Arai, MD

Department of Sports and Para-Sports Medicine, Graduate School of Medical Science, Kyoto Prefectural University of Medicine, 465 Kajiicho, Kawaramachi-Hirokoji, Kamigyo-ku, Kyoto 602-8566, Japan

Tel: +81-75-251-5549, Fax: +81-75-251-5841

E-mail: yarai89046@nike.eonet.ne.jp

This is an Open Access article distributed under the terms of the Creative Commons Attribution Non-Commercial License (http://creativecommons.org/licenses/by-nc/4.0/) which permits unrestricted non-commercial use, distribution, and reproduction in any medium, provided the original work is properly cited.
Although procedures for arthroscopic suture fixation of bony fragments have been described ${ }^{3}$, these procedures are technically complicated and anatomically unfamiliar even to experienced knee ligament surgeons. These methods include posteromedial, posterolateral, and trans-septal approaches. This report describes a patient with an avulsion fracture of the PCL from the tibia, in which the avulsed fragments were pulled out arthroscopically using three portals (anteromedial, anterolateral, and posteromedial portals) without special surgical instruments.

\section{Case Report}

A 46-year-old female who worked in the restaurant business was injured in a fall while riding a motorcycle. She complained of right knee pain and swelling, and was admitted to our hospital nine days after the injury. Her right knee showed patellar ballottement and a positive posterior drawer test, with this knee showing $>1 \mathrm{~cm}$ posterior sagging compared with the left knee. Radiographs and computed tomography (CT) images of the right knee showed a displaced avulsion fracture of the PCL tibial attachment (Fig. 1A and B). She was diagnosed with a PCL tibial attachment avulsion 

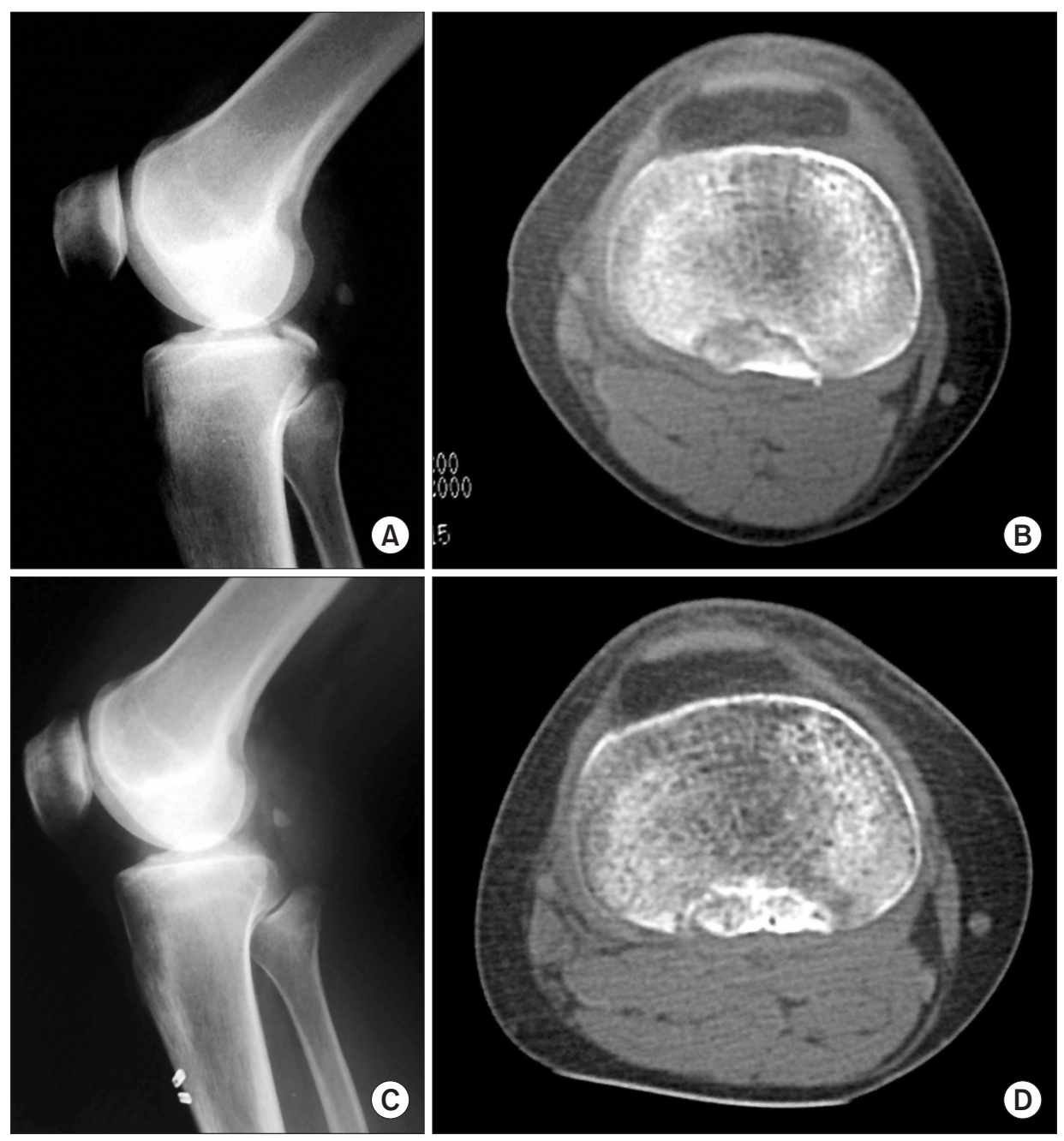

Fig. 1. (A) Preoperative X-ray showing a thin avulsed fragment of the posterior cruciate ligament arising from the tibial attachment. (B) Preoperative computed tomography (CT) showing that the avulsed fragment was thin and comminuted. (C) Xray taken three months after surgery showing that the fragment was well reduced to the tibial attachment and bone union was obtained. (D) Confirmation of bone union by CT three months after surgery.

fracture of the right knee and underwent arthroscopic osteosynthesis 13 days after the injury after providing informed consent.

\section{Operative Procedure}

The patient was placed in the supine position under general anesthesia. During the operation, the affected knee was set on the table at $90^{\circ}$ flexion with a leg holder (Zimmer, Warsaw, IN, USA), a position that relaxes the posterior structures and avoids neurovascular injury ${ }^{4)}$. Three arthroscopic portals (anterolateral, anteromedial, and posteromedial portals) were created for intraarticular surgery; to simplify surgery, a posterolateral portal was omitted. The joint was thoroughly examined through the anterolateral and anteromedial portals, and associated lesions were treated arthroscopically. There was no damage to the articular cartilage or substance of the PCL. The anteromedial portal was positioned just medial to the medial border of the patellar tendon to facilitate intercondylar access of the arthroscope to the posterior compartment. While viewing through the anterolateral portal, a blunt trocar in a sheath was gently inserted from the anteromedial portal, through the intercondylar notch, and into the posteromedial compartment. The trocar in the sheath was replaced with the arthroscope to visualize the posteromedial compartment. Under direct visualization, a spinal needle was inserted percutaneously into the posteromedial compartment at the joint level to ascertain the location and direction of the posteromedial portal. After making a small incision beside the needle, the posteromedial portal was established by expanding the portal with a straight inducer. To visualize the fragment accurately through the anteromedial and posteromedial portals, the sheaths inserted through these portals were kept in position during the operation. Under arthroscopic view from both portals, the avulsed fragment was carefully ascertained by the blunt trocar or a probe. Blood clots, free bony fragments, and soft tissue interposing between the fracture site and posterior capsule were debrided using a motorized shaver through the posteromedial portal until the avulsed fragment and tibial bony bed could be clearly released from the 
posterior capsule. Particular attention was paid to avoid injuring the PCL and bony fragment when shaving around the fragment. The medial wall of the posterior septum required partial shaving during the process, but entire perforation of the septum was not necessary. A 70 degree scope was not used in our patient because resection of the entire posterior septum was not necessary. The posterior septum has been reported to consist of two thin fibrous membranes, with a synovial membrane and intervening fatty tissue ${ }^{5)}$. The posterior septum is located laterally, allowing insertion of a Kirschner wire (K-wire) between the bony fragment and posterior septum by shaving the medial wall of the posterior septum. If the fracture does not extend to the lateral side of the tibia, the posterior septum can be dissected from the posteromedial portal. The synovial membrane between the anterior cruciate ligament (ACL) and PCL of the intercondylar fossa can be dissected by inserting an arthroscope between the ACL and PCL, making it possible to view the lateral side of the bone fragments. A 2-cm longitudinal incision was made just medial and 3 $\mathrm{cm}$ distal to the tibial tubercle. Under arthroscopic visualization from the anteromedial or posteromedial portal, a 2.0-mm K-wire was inserted through the anterior incision to a point just medial to the medial border of the anterior tibial bony bed (Fig. 2A). It was difficult to insert the tibial tunnel aiming device for the PCL because the PCL was present in the intercondylar fossa. Accurate insertion of the $\mathrm{K}$-wire requires careful preoperative measure- ments of the angle and distance of the K-wire from its puncture site point to the PCL attachment. As per specific preoperative planning, we used CT or magnetic resonance imaging. By using a sagittal image wherein the PCL attachment of tibia was revealed, we measured the distance from the tibial articular surface to the insertion point of the K-wire. In addition, we measured the insertion angle between the K-wire and the anterior cortex of tibia. Damage to the posterior neurovascular bundle can be avoided by not inserting the K-wire at a greater distance. A blunt rod was inserted from the anteromedial portal toward the PCL attachment portion, and the arthroscope was inserted from the posteromedial portal. It is important to insert the K-wire towards the point of intersection of the blunt rod from the anteromedial portal and the arthroscope from the posteromedial portal. In particular, the direction of the blunt rod became the rotational reference for the K-wire insertion.

Care was taken to prevent the K-wire from penetrating the fragment or center of the bed. A $0.55-\mathrm{mm}$ suture wire was folded to make a loop and introduced into the posteromedial compartment via the bone tunnel. The same procedure was repeated for the lateral side of the bony bed. Thus, two loops of suture wire were inserted into the posteromedial compartment (Fig. 2B). The loops were opened with forceps in the posteromedial compartment. A 16-gauge needle was inserted from the posteromedial portal, through the medial and lateral loop wires, and into the postero-
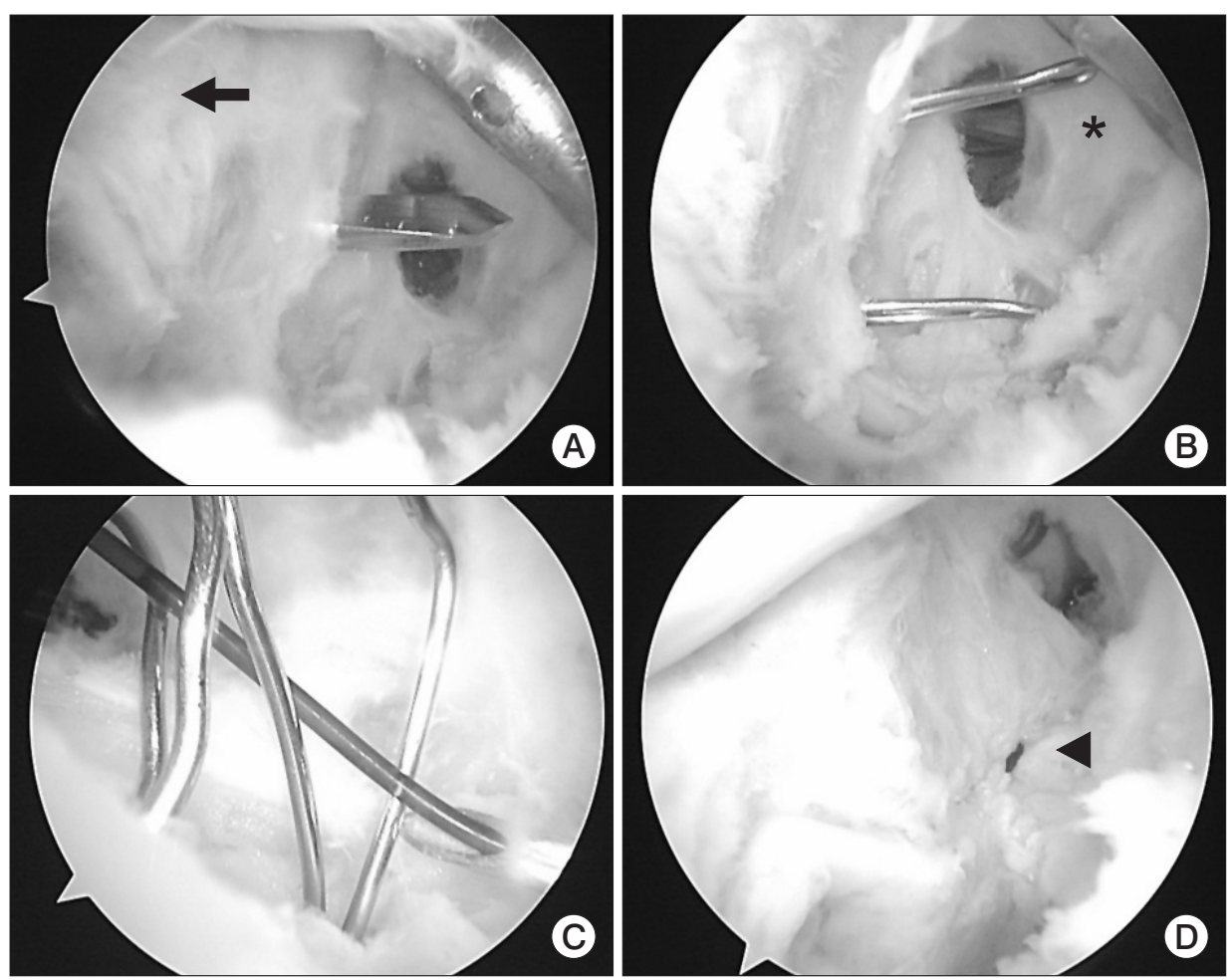

Fig. 2. (A) Posteromedial view. A tibial bone tunnel was made on the medial side of the bony bed with a $2.0 \mathrm{~mm}$ Kirschner wire. The wire was inserted from the anterior aspect of the tibia. Arrow: posterior cruciate ligament. (B) Posteromedial view. A $0.55-\mathrm{mm}$ suture wire was folded to make loops and inserted into the posteromedial compartment via the bone tunnel. Two loops were inserted. Asterisk: posterior septum. (C) Anteromedial view. The loops of suture wires were opened with forceps inserted into the posteromedial compartment through the posteromedial portal, and FiberWire (Arthrex) was passed through the loops. (D) The lateral and medial loop wires were pulled outside and the avulsion fragment was fixed with FiberWire on the tibia by providing tension using an Endobutton (Smith \& Nephew) device. Arrowhead: fixation thread. 
medial compartment. A 2-0 nylon thread was passed through the needle, and approximately $5 \mathrm{~cm}$ of the thread was inserted into the posteromedial compartment (Fig. 2C). The nylon thread was relayed to the 2-0 FiberWire (Arthrex, Naples, FL, USA), and the lateral and medial loop wires attached to the thread were pulled to the outside (Fig. 2D). The thread was fixed onto the tibia using an Endobutton fixation device (Smith \& Nephew, Andover, MA, USA) while applying anterior drawer force to the proximal tibia with the knee flexed at $90^{\circ}$, without cutting the cortical bridge between the bone tunnels (Fig. 3). Another fixation thread was placed on the posterior side of the bony bed and fixed in the same manner, resulting in two fixation threads being placed over the bony fragment. After tying the threads, reduction and fixation of the fragment were confirmed arthroscopically.

\section{Postoperative Care and Assessment}

A plaster cast was applied for 2 weeks with the knee in the extended position. Isometric exercises to strengthen the quadriceps muscle were started 2 days postoperatively. Partial weight bearing was started with an articulated hard brace placed on the knee,

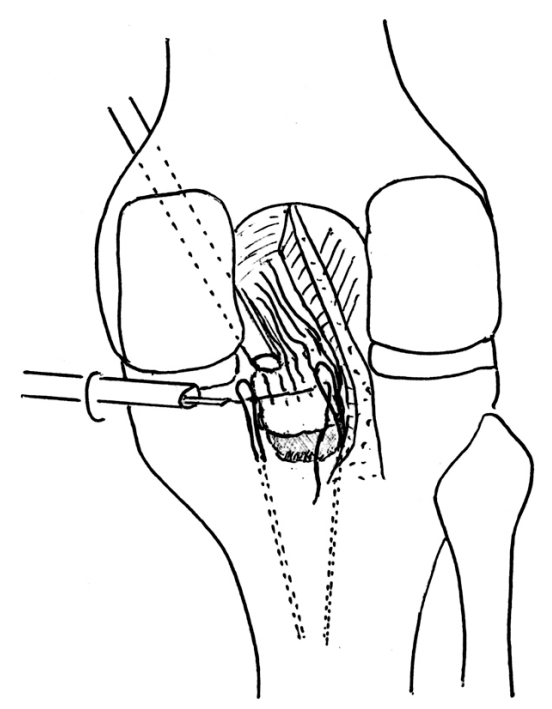

Fig. 3. Schematic posterior view of the procedure. Under arthroscopic visualization from the anteromedial portal, the adhesions of the avulsed fragment to the posterior capsule and the medial wall of the posterior septum were debrided using a motorized shaver through the posteromedial portal. The medial and lateral loops of the $0.55-\mathrm{mm}$ suture wire inserted into the posteromedial compartment via bone tunnels made by a 2.0-mm Kirschner wire were opened by forceps. Fixation thread was passed through a 16-gauge needle inserted into the two loops. The two loop wires attached to the thread were pulled outside, and the thread was fixed on the anterior aspect of the tibia. In this operation involving only the posteromedial compartment, the fragment was reduced and fixed tightly to the bony bed. and 3 weeks were required for full weight bearing. Range of motion exercises in $0^{\circ}-90^{\circ}$ knee flexion were started 2 weeks after surgery for 3 weeks. Subsequently, a maximum flexion of $120^{\circ}$ was permitted until 10 weeks after surgery.

Three months after the operation, bone union was confirmed by X-ray and CT imaging (Fig. 1C and D). The patient returned to her job without any sequelae. Eighteen months after the operation, she had no limit in range of motion and no posterior sagging of the right knee.

\section{Discussion}

Treatment of fresh avulsion fractures of the PCL depends on the degree of avulsion fracture displacement. Conservative therapy is generally performed if the degree of avulsion fracture displacement is mild, whereas surgery is performed in patients with marked displacement and tibial posterior instability ${ }^{6}$. Because the PCL is important in rollback of the femur during knee flexion, for posterior stability of the knee, and for lower leg screw home rotation, PCL dysfunction may result in degenerative changes to the knee joint. Because surgery is believed to yield better outcomes than conservative therapy, surgery has been recommended even for patients with relatively mild displacement. Invasive fixation that markedly opens the back of the knee was commonly performed in the past. Recent developments in instruments and techniques for arthroscopic surgery have resulted in the addition of arthroscopic treatment conditions such as injuries to the meniscus and cruciate ligaments, and osteochondritis dissecans. Arthroscopic surgery has also been used to treat avulsion fractures of the ACL, and we previously reported a technique for arthroscopic pullout fixation ${ }^{7)}$. The ability of arthroscopic surgery to treat avulsion fractures of the PCL can lead to the simultaneous treatment of accompanying intraarticular lesions using a minimally invasive procedure, while reducing the risk of damage to soft tissue and neurovascular bundles in the back of the knee. The first study of arthroscopic fixation using a special forceps guide on avulsion fractures of the PCL was successful in six of eight cadaveric knees ${ }^{8}$. Subsequently, arthroscopic fixation was performed using a $4.5-\mathrm{mm}$ cannulated screw ${ }^{9}$, followed by the use of screws, K-wires, and suture wires/threads.

Large bone fragments can be reduced and fixed with screws or K-wires in an anterograde or retrograde manner, or using a suture anchor. Direct fixation of small and thin or comminuted fragments to the bone bed, however, is difficult, not allowing the suture wire/thread to be pulled out to the anterior side of the tibia for reduction and fixation of the fragment ${ }^{3)}$. In particular, 
arthroscopic surgery using a pullout procedure requires an accurate examination and visualization of the fragment and bone bed as well as pulling out of the fixation wire/thread from the posterior to the anterior side of the fragment. Several posteromedial portals have been used for this complicated procedure. Furthermore, an excellent arthroscopic view was reportedly obtained by inserting through a posterolateral portal and dissecting the posterior septum that separates the posterior joint space into medial and lateral compartments to use as a trans-septal portal ${ }^{3)}$. The posterior septum consists of two layers of thin fibrous membranes with synovium and fat tissue between them. Although the middle genicular artery runs through the upper margin of the posterior septum, no important tissue is present in the central region $^{5}$. However, as vascularization varies greatly among individuals, the creation of posterolateral and trans-septal portals can damage neurovascular bundles. A recent study of arthroscopic fixation using a suspensory fixation device required only three portals, an anteromedial, an anterolateral, and a posteromedial portal $^{10)}$. However, this method required drilling a 4-mm hole in the tibia, which may result in breakage of the bone fragment of PCL attachment. We therefore developed an arthroscopic fixation technique for avulsion fracture of the PCL with a small or comminuted fragment that requires drilling a 2-mm drill hole in the tibia. This surgical procedure only involved the posteromedial compartment, which can be approached from the posteromedial portal with minimal resection of the posterior septum. This technique does not require any special instruments or removal of any device for fixation of the fragment from the joint, even after bone union. This surgical procedure is thus convenient and less invasive than those reported previously. This study described a patient with an avulsion fracture of the PCL from the tibia treated by pulling out the avulsed fragments arthroscopically. This surgical procedure was convenient and less invasive than previous methods, requiring no special surgical instruments.

\section{Conflict of Interest}

No potential conflict of interest relevant to this article was reported.

\section{References}

1. Lee BK, Nam SW. Rupture of posterior cruciate ligament: diagnosis and treatment principles. Knee Surg Relat Res. 2011; 23:135-41.

2. Torisu T. Isolated avulsion fracture of the tibial attachment of the posterior cruciate ligament. J Bone Joint Surg Am. 1977;59:68-72.

3. Kim SJ, Shin SJ, Choi NH, Cho SK. Arthroscopically assisted treatment of avulsion fractures of the posterior cruciate ligament from the tibia. J Bone Joint Surg Am. 2001;83:698-708.

4. Matava MJ, Sethi NS, Totty WG. Proximity of the posterior cruciate ligament insertion to the popliteal artery as a function of the knee flexion angle: implications for posterior cruciate ligament reconstruction. Arthroscopy. 2000;16:796804.

5. Ahn JH, Ha CW. Posterior trans-septal portal for arthroscopic surgery of the knee joint. Arthroscopy. 2000;16:774-9.

6. Sasaki SU, da Mota e Albuquerque RF, Amatuzzi MM, Pereira CA. Open screw fixation versus arthroscopic suture fixation of tibial posterior cruciate ligament avulsion injuries: a mechanical comparison. Arthroscopy. 2007;23:122630.

7. Hara K, Kubo T, Shimizu C, Suginoshita T, Hirasawa Y. Arthroscopic reduction and fixation of avulsion fracture of the tibial attachment of the anterior cruciate ligament. Arthroscopy. 2001;17:1003-6.

8. Martinez-Moreno JL, Blanco-Blanco E. Avulsion fractures of the posterior cruciate ligament of the knee: an experimental percutaneous rigid fixation technique under arthroscopic control. Clin Orthop Relat Res. 1988;(237):204-8.

9. Littlejohn SG, Geissler WB. Arthroscopic repair of a posterior cruciate ligament avulsion. Arthroscopy. 1995;11:235-8.

10. Gwinner C, Kopf S, Hoburg A, Haas NP, Jung TM. Arthroscopic treatment of acute tibial avulsion fracture of the posterior cruciate ligament using the tightrope fixation device. Arthrosc Tech. 2014;3:e377-82. 